\title{
GRB 110715A: MULTIWAVELENGTH STUDY OF THE FIRST GAMMA-RAY BURST OBSERVED WITH ALMA
}

\author{
R. Sánchez-Ramírez ${ }^{1}$, P. Hancock ${ }^{2}$, T. Murphy $^{2}$, A. de Ugarte Postigo ${ }^{1,3}$, \\ J. Gorosabel ${ }^{1,4,5}$, D.A. Kann ${ }^{6,7}$, C.C. Thöne ${ }^{1}$, A. Lundgren ${ }^{8,9}$, A. Kamble ${ }^{10}$, \\ S.R. Oates ${ }^{11}$, J.P.U. Fynbo ${ }^{3}$, I. de Gregorio Monsalvo ${ }^{8,9}$, D. Garcia-Appadoo ${ }^{8,9}$, \\ S. Martín ${ }^{8}$, N.P.M. Kuin ${ }^{11}$, J. Greiner ${ }^{7}$ and A.J. Castro-Tirado ${ }^{1}$
}

\begin{abstract}
GRB 110715A had a bright afterglow that was obscured in the optical by a high Galactic extinction. We discovered the submillimeter counterpart with APEX and followed it in radio with ATCA for over 2 months. Additional submm observations were performed with ALMA as a test of the ToO procedures during commissioning, becoming the first GRB afterglow to be detected by the observatory. UV, optical and NIR observations were performed with Swift/UVOT and $2.2 \mathrm{~m} /$ GROND in La Silla and X-ray data were obtained by Swift/XRT. The dataset is complemented with spectroscopic data from the VLT/X-shooter spectrograph. The absorption features present in the intermediate resolution optical/nIR spectra reveal a redshift of 0.8224 and a host galaxy environment with low ionization. We fit in the host galaxy absorption features two velocity components separated by $30 \mathrm{~km} / \mathrm{s}$, implying a host galaxy with low dynamical activity.
\end{abstract}

\footnotetext{
1 Instituto de Astrofísica de Andalucía, IAA-CSIC, Glorieta de la Astronomía s/n, 18008, Spain

2 Sydney Institute for Astronomy, School of Physics, The University of Sydney, NSW 2006, Australia

${ }^{3}$ Dark Cosmology Centre, Niels Bohr Institute, Juliane Maries Vej 30, 2100 Copenhagen $\varnothing$, Danemark

4 Unidad Asociada Grupo Ciencia Planetarias UPV/EHU-IAA/CSIC, Departamento de Física Aplicada I, E.T.S. Ingeniería, Universidad del País Vasco UPV/EHU, Alameda de Urquijo s/n, 48013 Bilbao, Spain

${ }^{5}$ Ikerbasque, Basque Foundation for Science, Alameda de Urquijo 36-5, 48008 Bilbao, Spain

6 Thüringer Landessternwarte Tautenburg, Sternwarte 5, 07778 Tautenburg, Germany

7 MPI für extraterrestrische Physik, 85740 Garching, Germany

8 European Southern Observatory, Vitacura Casilla 19001, Santiago de Chile 19, Chile

9 Joint ALMA Observatory, Alonso de Córdova 3107, Vitacura, Santiago, Chile

10 Harvard-Smithsonian Center for Astrophysics, 60 Garden St., Cambridge, MA 02138, USA

11 Mullard Space Science Laboratory, University College London, Holmbury St. Mary, Dorking, Surrey RH5 6NT, UK
} 


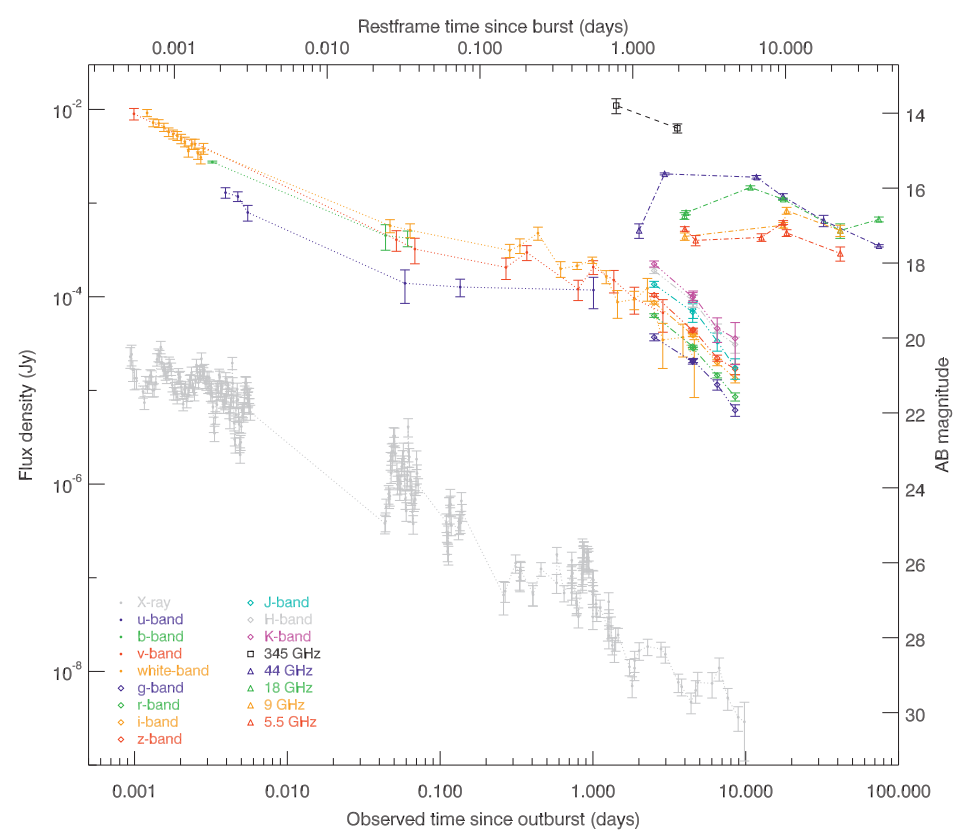

Fig. 1. Afterglow light curve in the 17 observed bands.

\section{Observations}

GRB 110715A (Sonbas et al. 2011) was an intense burst, discovered by the Burst Alert Telescope (BAT) onboard the Swift satellite. It was classified as a long burst, with a $T_{90} \sim 13 \mathrm{~s}$.

The burst happened at a galactic latitude of only 6 degrees, implying that it was optically obscured by dust from the Milky Way $\left(E_{(B-V)}=0.59\right)$, which complicated the optical follow-up. In spite of this, it was detected by the Ultra Violet/Optical Telescope (UVOT) on Swift just a few minutes after the gamma emission, providing us the unique optical dataset of the early times. This implied that the intrinsic luminosity of the event was high. In view of this, we triggered our target of opportunity programme at the $12 \mathrm{~m}$ Atacama Pathfinder EXperiment (APEX), using the LABOCA instrument to observe at $850 \mu \mathrm{m}$. Observations were performed 1.42 days after the GRB onset, and we discovered a bright submillimetre counterpart, with an intensity of $10.4 \pm 2.4 \mathrm{mJy}$ (de Ugarte Postigo et al. 2011), making it the fourth brightest GRB ever observed at these wavelengths (de Ugarte Postigo et al. 2012a).

As a test of the target of opportunity procedure, GRB 110715A was subsequently observed at Atacama Large Millimeter Array (ALMA), in what became the first observation of a GRB by this observatory.

Following the detection of the afterglow at submm wavelengths with APEX, radio observations were obtained with the Australia Telescope Compact Array 
(ATCA) two and three days after the outburst. These observations resulted in further detections of the afterglow at $44 \mathrm{GHz}$ (Hancock et al. 2011). Observations were obtained up to 75 days post burst at 44, 18, 9, and $5 \mathrm{GHz}$.

We obtained follow-up observations of the optical/NIR afterglow of GRB 110715A with the seven-channel imager GROND (Greiner et al. 2008) mounted on the $2.2 \mathrm{~m} \mathrm{MPI/ESO} \mathrm{telescope} \mathrm{stationed} \mathrm{in} \mathrm{La} \mathrm{Silla,} \mathrm{Chile.} \mathrm{The}$ afterglow is detected in all $g, r, i, z, J, H$ and $K$ filters between 2.5 and 8 days.

For the study of the X-ray emission, we made use of the Swift/XRT publicly available data (Evans et al. 2009). All the photometric observations are compiled in the form of a multi-band light curve in Figure 1.

The dataset is completed with a spectrum obtained with the X-shooter spectrograph at the Very Large Telescope, in Paranal Observatory (Chile), 12.7 hours after the GRB. The spectrum covers the complete range between 3000 and $24800 \AA$, although the strong optical extinction only allows detection of the continuum above $\sim 3600 \AA$.

\section{Preliminary results}

The ALMA Science Team reported a preliminary detection of this source of $4.9 \pm$ $0.6 \mathrm{mJy}$ at $850 \mu \mathrm{m}$ after only $25 \mathrm{mins}$ on source with 7 antennas 3.6 days after the onset. In spite of this, it is the deepest observation of a GRB at this band up to date. The centroid of the ALMA position is found at equatorial coordinates (J2000), RA: 15:50:44.05, Dec: -46:14:06.5 with an uncertainty of $0 . \prime 3 \times 0$ "' 1 at a position angle of 76 degrees, which is the most precise localisation of the event. We detect absorption lines in the complete X-shooter spectrum that we identify as FeII*/FeII, MgI, MgII, CaI and CaII at a common redshift of $0.8224 \pm 0.0002$. We have measured equivalent widths of these lines and limits for several others using a self-developed code. Following the prescription of de Ugarte Postigo et al. (2012b), we obtain a line strength parameter for GRB 110715A of $L S P=-0.42$, implying that this event is in the percentile 29 of line strengths, indicating a lower than average column density of material in the line of sight. This can point towards a small host galaxy. This is consistent with the fact that there are two velocity components in the absorption features spanning only $30 \mathrm{~km} \mathrm{~s}^{-1}$, also lower than average.

\section{References}

de Ugarte Postigo, A., Lundgren, A., Mac-Auliffe, F., et al., 2011, GCN 12168

de Ugarte Postigo, A., Lundgren, A., Martín, S., et al., 2012, A\&A, 538, A44

de Ugarte Postigo, A., Fynbo, J.P.U., Thoene, C.C., et al., 2012, A\&A, 548, A11

Evans, P.A., Beardmore, A.P., Page, K.L., et al., 2009, MNRAS, 397, 1177

Hancock, P.J., Murphy, T., \& Schmidt, B.P., 2011, GCN 12171

Sonbas, E., Barthelmy, S.D., Baumgartner, W.H., et al., 2011, GCN 12158 
